\title{
Structural Studies of Polyesters. VI. Disordered Crystal Structure (Form II) of Poly( $\beta$-propiolactone)
}

\author{
Kazuaki Suehiro, ${ }^{*}$ Yozo Chatani, and Hiroyuki Tadokoro \\ Department of Polymer Science, Faculty of Science, Osaka \\ University, Toyonaka, Osaka 560, Japan.
}

(Received October 5, 1974)

\begin{abstract}
The crystal structure of a modification (Form II) of poly( $\beta$-propiolactone) was examined by $\mathrm{X}$-ray diffraction. This modification shows a unique $\mathrm{X}$-ray fiber pattern, i.e., discrete reflections on the equatorial line and continuous scatterings on each layer line, which is characteristic of a longitudinal disordered crystal structure. Conventional cell dimensions are $a^{\prime}=7.73, b^{\prime}=4.48, c$ (fiber axis) $=4.77 \AA$, and $\gamma^{\prime}=90^{\circ}$. The unit cell contains two planar zigzag chains. The molecular chains pack side-by-side in a regular fashion forming a sheet structure parallel to the $a^{\prime} c$ plane. However, the mutual levels of the chains along the fiber axis are irregular, i.e., the crystal of Form II is in a paracrystalline state.
\end{abstract}

KEY WORDS Poly( $\beta$-propiolactone) / Aliphatic Polyester / X-Ray Diffraction / Crystal Structure / Disordered Structure / Small Angle X-Ray Diffraction /

The crystal structures of several lower member polyesters of the type $\left[-\mathrm{O}-\left(\mathrm{CH}_{2}\right)_{x}-\mathrm{O}-\mathrm{CO}-\right.$ $\left.\left(\mathrm{CH}_{2}\right)_{y}-\mathrm{CO}-\right]_{n}$ and $\left[-\left(\mathrm{CH}_{2}\right)_{z}-\mathrm{CO}-\mathrm{O}-\right]_{n}$ and of their derivatives have been revealed in our laboratory: polyethylene oxalate, ${ }^{1}$ polyethylene succinate, ${ }^{1}$ polytetramethylene succinate, ${ }^{2}$ polyglicolide ${ }^{3}$ poly $(\beta$-hydroxybutylate $),{ }^{4}$ and $\operatorname{poly}(\beta$ ethyl- $\beta$-propiolactone). ${ }^{5} \quad$ These lower member polyesters are interesting from the viewpoint of molecular and crystal structures, because their structures are quite different from higher member polyesters $^{6,7}$ which have essentially polyethylenelike molecular and/or crystal structures. $\operatorname{Poly}(\beta-$ propiolactone), the second member of the series $\left[-\left(\mathrm{CH}_{2}\right)_{z}-\mathrm{CO}-\mathrm{O}-\right]_{n}$, has three crystalline modifications (in addition to the two modifications reported by Okamura, et al., ${ }^{8}$ the third one has been found by us $^{9}$ ). One of these modifications shows a unique X-ray fiber pattern, i.e., discrete reflections on the equatorial line and diffuse continuous scatterings on the layer lines. This modification has been called Form II. Wasai, et al. ${ }^{10}$ called this modification $\beta$-form and proposed a planar zigzag chain structure for the molecular conformation in this modifi-

\footnotetext{
* Present address: Faculty of Science and Engineering, Saga University.
}

cation on the basis of the observed fiber period. They also speculated on a disordered packing of the molecular chains in the crystal lattice with random displacement along the fiber axis. The present paper deals with the crystal structure of Form II in more detail.

\section{EXPERIMENTAL}

\section{Samples}

Two differently originated samples were used in the present study. One was prepared by polymerization of $\beta$-propiolactone with an ionic catalyst, and the other was prepared by $\gamma$-irradiated solid-state polymerization of $\beta$-propiolactone. Uniaxially oriented samples of Form II were obtained by slow cooling of molten samples followed by cold drawing to about five times its original length. Poly( $\beta$-propiolactone) obtained by the solid-state polymerization of a large single crystal of $\beta$-propiolactone gave directly a well oriented X-ray fiber pattern of Form II as shown in Figure 1. The present study was therefore carried out mainly with the latter sample.

\section{$X$-Ray Measurement \\ $\mathrm{X}$-ray diffraction patterns were taken with}




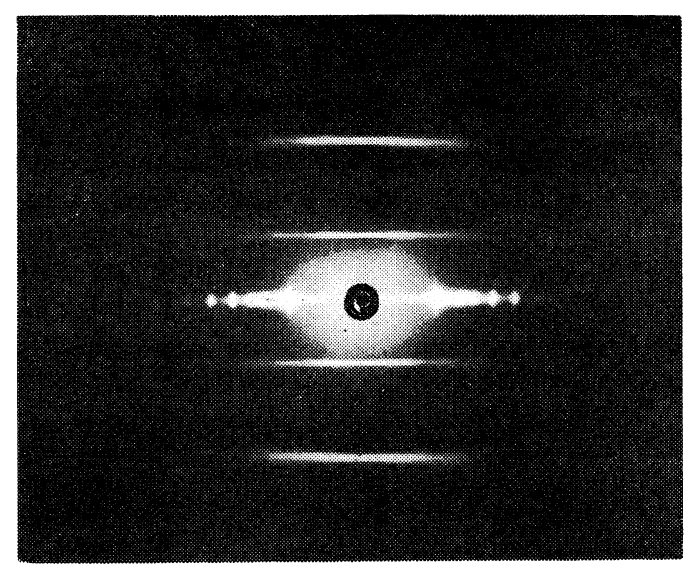

Figure 1. X-ray fiber photograph of Form II of poly( $\beta$-propiolactone) which is as-polymerized by the solid-state polymerization.

nickel-filtered $\mathrm{CuK} \alpha$ radiation. In order to examine whether continuous scatterings on the equatorial line exist or not, the $\mathrm{CuK} \alpha$ radiation
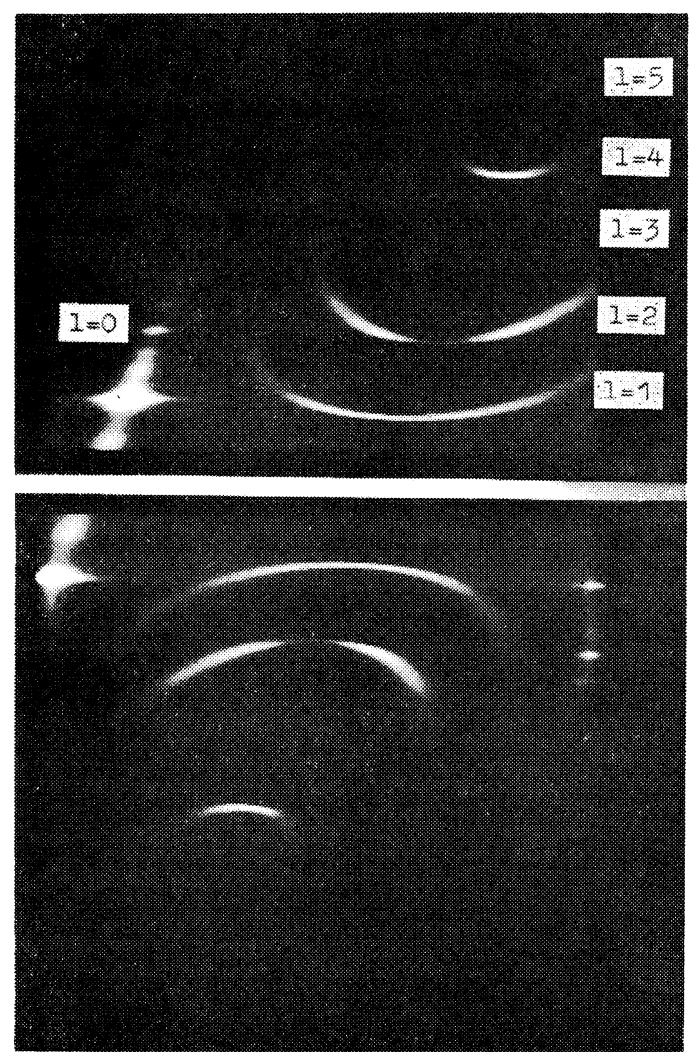

Figure 2. Weissenberg photograph of Form II of poly $(\beta$-propiolactone). monochromatized with a LiF single crystal was also used; no continuous scatterings were observed in this photograph. The intensities of equatorial reflections were measured by a single crystal diffractometer in addition to visual estimation from the photographs. The scattering intensities of the layer lines were measured by a microdensitometer for Weissenberg photographs which were taken by oscillating the uniaxial sample about an axis perpendicular to the fiber axis (Figure 2).

\section{STRUCTURE DETERMINATION}

The characteristic feature of the X-ray fiber pattern of Form II is discrete reflections on the equatorial line and streaks on every layer line. This feature could be explained by a disordered crystal structure in which the molecular chains themselves have a periodic conformation and also in which the packing of the chains in the lateral direction with respect to the fiber axis is periodic, while the mutual levels of the chains in the fiber direction are irregular.

In order to explain the intensity distribution of the X-ray fiber pattern for the disordered structure mentioned above, an intensity formula was introduced. If the chains align in the $c$ direction, the intensity at a position $\boldsymbol{S}(|\boldsymbol{S}|=$ $2 \sin \theta(\lambda)$ in the reciprocal space is given as follows (see Appendix):

$$
\begin{aligned}
I(\boldsymbol{S})= & {\left[\left|\sum_{m} F_{m}(\boldsymbol{S})\right|^{2} \frac{\sin ^{2} \pi N_{1} s_{1} a \sin ^{2} \pi N_{2} s_{2} b}{\sin ^{2} \pi s_{1} a \sin ^{2} \pi s_{2} b}\right.} \\
& \times \frac{\sin ^{2} \pi N_{3} s_{3} c}{\left(\pi s_{3} c\right)^{2}}+N_{1} N_{2} \sum_{m}\left|F_{m}(S)\right|^{2} \\
& \left.\times\left\{1-\frac{\sin ^{2} \pi s_{3} c}{\left(\pi s_{3} c\right)^{2}}\right\}\right] \frac{\sin ^{2} \pi N_{3} s_{3} c}{\sin ^{2} \pi s_{3} c}
\end{aligned}
$$

where $F_{m}(S)$ is the structure factor of the chain of $m$ th state. $N_{1}, N_{2}$, and $N_{3}$ are the number of repeats along the $a, b$, and $c$ axes, respectively, and $s_{1}, s_{2}$, and $s_{3}$ are the components of $S$ to the three reciprocal axes. This formula assumes that the diffraction intensities are negligible when $s_{3} c \neq l(l$; any integer), i.e., intensities distribute only on layers and, in the first term of right side of eq $1, s_{1} a=h$ and $s_{2} b=k$ ( $h$ and $k$; integer) when $N_{1}$ and $N_{2}$ are large. Therefore, eq 1 becomes 
$I(S)=N_{1}{ }^{2}{N_{2}}^{2} N_{3}{ }^{2}\left|\sum_{m} F_{m}(h k 0)\right|^{2} \quad$ for $\quad l=0$

and

$I(\boldsymbol{S})=N_{1} N_{2} N_{3}^{2} \sum_{m}\left|F_{m}\left(s_{1} s_{2} l\right)\right|^{2} \quad$ for $\quad l \neq 0$

Equations 2 and 3 indicate that the equatorial reflections are discrete, while the reflections on the layer lines are continuous.

As the sample used for the intensity measurement was uniaxially oriented, in order to make correspondence to the observed intensities it is necessary to average the calculated intensities over the azimuthal angle $\Psi$ about the $c$ axis. Using cylindrical coordinates $(R, \Psi, l / c)$ in the reciprocal space $\left(|S|^{2}=\left(R^{2}+l^{2} / c^{2}\right)^{1 / 2}\right)$, and ignoring constants, the cylindrically averaged intensity of the layer line $l$ is expressed as follows:

$$
\langle I(S)\rangle_{\Psi}=I(R, l / c)=\frac{1}{2 \pi} \sum_{m} \int_{0}^{2 \pi}\left|F_{m}(R, \Psi, l / c)\right|^{2} \mathrm{~d} \Psi
$$

As the integration in eq 4 is independent of $m$, eq 4 is given by ${ }^{7}$

$$
\begin{gathered}
I(R, l / c)=|T|^{2}=\sum_{j} \sum_{j^{\prime}} f_{j} f_{j^{\prime}} J_{0}\left(2 \pi R r_{j j^{\prime}}\right) \\
\times \exp \left[2 \pi \mathrm{i} l\left(z_{j}-z_{j^{\prime}}\right) / c\right] \\
r_{j j^{\prime}}^{2}=\left(x_{j}-x_{j^{\prime}}\right)^{2}+\left(y_{j}-y_{j^{\prime}}\right)^{2}
\end{gathered}
$$

$T$ is the so-called "molecular transform". $J_{0}$ is a Bessel function of zero order, and $f_{j}$ and $\left(x_{j}, y_{j}, z_{j}\right)$ are the atomic scattering factor and atomic coordinates of the $j$ th atom, respectively. The summation in eq 5 is taken for all atom pairs in the repeating unit. The calculated intensities are then multiplied by an anisotropic thermal factor $\exp \left\{-\frac{1}{2}\left[B_{\mathrm{r}} R^{2}+B_{1}(l / c)^{2}\right]\right\}$, where $B_{\mathrm{r}}$ and $B_{1}$ are the thermal coefficients in the directions perpendicular and parallel to the $c$ axis, respectively.

The identity period along the fiber axis was found to be $4.77 \AA$ from the interval of the layer lines. From the equatorial reflections, an orthogonal cell having the cell dimensions of $a^{\prime}=$ $7.73, b^{\prime}=4.48 \AA$, and $\gamma^{\prime}=90^{\circ}$ was conventionally determined. The $c$ dimension of $4.77 \AA$ indicates that the molecular conformation of Form II is essentially planar zigzag. The observed density measured by the flotation method was $1.38 \mathrm{~g} / \mathrm{cm}^{3}$. Therefore, two molecular chains pass through the unit cell: the calculated density of $1.43 \mathrm{~g} / \mathrm{cm}^{3}$ is reasonable compared with the observed value.

Since the reflections on the layer lines are not spotty and a few equatorial reflections are superimposed with reflections of different indicies, neither the space group nor the plane group could be decided uniquely. Trial and error procedures using the equatorial reflections were therefore adopted to determine the molecular arrangement projected on to the $a^{\prime} b^{\prime}$ plane. A

Table I. Fractional atomic coordinates ${ }^{a}$

\begin{tabular}{cccc}
\hline Atom & $x$ & $y$ & $z$ \\
\hline $\mathrm{C}(1)$ & 0.238 & 0.250 & 0.000 \\
$\mathrm{C}(2)$ & 0.236 & 0.250 & 0.478 \\
$\mathrm{C}(3)$ & 0.351 & 0.250 & 0.738 \\
$\mathrm{O}(1)$ & 0.341 & 0.250 & 0.233 \\
$\mathrm{O}(2)$ & 0.078 & 0.250 & 0.000 \\
$\mathrm{H}(1)$ & 0.155 & 0.052 & 0.478 \\
$\mathrm{H}(2)$ & 0.155 & 0.448 & 0.478 \\
$\mathrm{H}(3)$ & 0.432 & 0.052 & 0.738 \\
$\mathrm{H}(4)$ & 0.432 & 0.448 & 0.738 \\
\hline
\end{tabular}

a The $x$ and $y$ coordinates of the second molecular chain in the unit cell are given by $1-x$ and $\frac{1}{2}+y$. The $z$ coordinates are tentative values as described in the text.

\begin{tabular}{|c|c|c|c|c|c|}
\hline$h k l$ & $d_{0}, \AA$ & $d_{\mathrm{c}}, \AA$ & $\sqrt{I_{0}}{ }^{a}$ & $\sqrt{\bar{I}_{\mathrm{c}}}{ }^{\mathrm{a}}$ & $F$ \\
\hline 100 & - & 7.73 & - & 1 & 0.8 \\
\hline $\left.\begin{array}{lll}1 & 1 & 0 \\
2 & 0 & 0\end{array}\right\}$ & 3.88 & $\begin{array}{l}3.88 \\
3.87\end{array}$ & 80 & 80 & $\left\{\begin{array}{l}38.3 \\
15.6\end{array}\right.$ \\
\hline 210 & 2.92 & 2.93 & 14 & 9 & 4.4 \\
\hline 300 & 2.58 & 2.58 & 13 & 13 & 9.2 \\
\hline $\left.\begin{array}{lll}0 & 2 & 0 \\
3 & 1 & 0\end{array}\right\}$ & 2.25 & $\begin{array}{l}2.24 \\
2.23\end{array}$ & 24 & 33 & $\left\{\begin{array}{r}23.2 \\
1.2\end{array}\right.$ \\
\hline 120 & - & 2.15 & - & 1 & 0.7 \\
\hline $\left.\begin{array}{lll}2 & 2 & 0 \\
4 & 0 & 0\end{array}\right\}$ & 1.93 & $\begin{array}{l}1.94 \\
1.93\end{array}$ & 16 & 13 & $\left\{\begin{array}{l}6.2 \\
2.4\end{array}\right.$ \\
\hline 410 & - & 1.77 & - & 12 & 6.2 \\
\hline 320 & 1.67 & 1.69 & 12 & 10 & 5.2 \\
\hline 500 & - & 1.55 & vvw & 2 & 1.2 \\
\hline $\left.\begin{array}{lll}1 & 3 & 0 \\
4 & 2 & 0 \\
5 & 1 & 0\end{array}\right)$ & 1.46 & $\begin{array}{l}1.47 \\
1.46 \\
1.46\end{array}$ & 12 & 13 & $\left\{\begin{array}{l}6.6 \\
0.7 \\
0.4\end{array}\right.$ \\
\hline 230 & - & 1.39 & - & 2 & 0.9 \\
\hline 330 & - & 1.29 & - & 0 & 0.0 \\
\hline 600 & - & 1.29 & - & 1 & 0.9 \\
\hline 520 & - & 1.27 & - & 1 & 0.8 \\
\hline
\end{tabular}

Table II. Comparison between observed and calculated spacings and structure factors of $h k 0$ reflections

a $\sqrt{I}=\sqrt{\sum m_{i} F_{i}^{2}} ; m$, multiplicity of reflection. 
good agreement between observed and calculated intensities for the $h k 0$ reflections was achieved when the plane group $p g$ and the atomic coordinates listed in Table I were adopted. A comparison between the observed and calculated intensities for the $h k 0$ reflections is given in Table II. For the layer lines, the calculation of eq 5 was carried out by using the atomic coordinates in Table I, where $z$ coordinates were obtained from an assumption that the planar

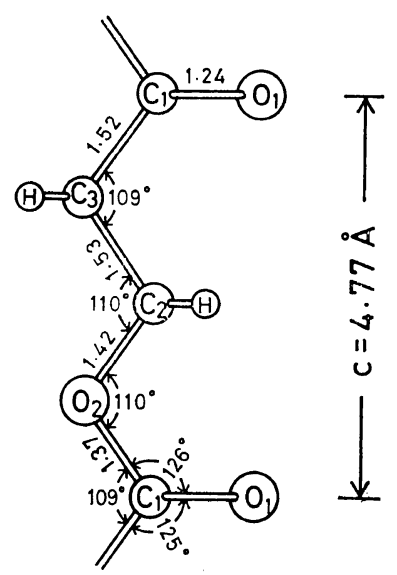

Figure 3. Dimensions of molecular chain in Form II of $\operatorname{poly}(\beta$-propiolactone).

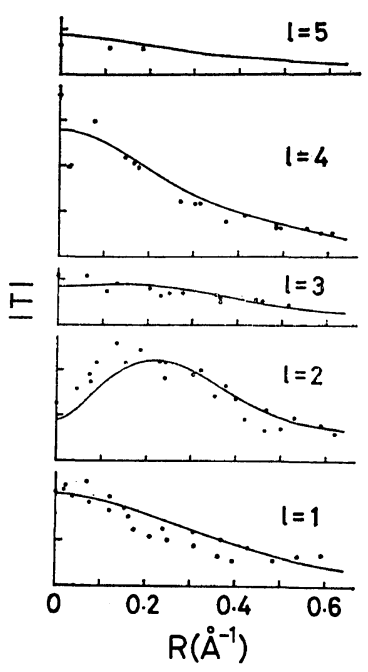

Figure 4. Comparison between observed and calculated structure factors $|T|$ for layer lines. Solid lines are calculated values and points are observed ones. The observed values are corrected for polarization factor. chain of poly( $\beta$-propiolactone) has the molecular dimensions as shown in Figure 3. The observed and calculated structure factor $|T|$ is shown in Figure 4. In the structure factor calculation, thermal coefficients of $B_{\mathrm{r}}=10 \AA^{2}$ and $B_{1}=1.5 \AA^{2}$ were used. The intensities of the 1st, 2nd, and 4th layer lines are strong, while those of the 3rd and 5th layer lines are weak. Viewing the intensity distribution on each layer line, the maximum intensity appears at the meridian $(R=0)$ on the 1st and 4th layer lines, while on the 2 nd layer line the intensity is minimum at the meridian. As a whole, the calculated intensities are in good agreement with the observed ones, though the observed values are somewhat scattered.

\section{RESULTS AND DISCUSSION}

The molecular arrangement viewed along the fiber axis is illustrated in Figure 5. The molecular chains are arranged side-by-side in a regular fashion. The chains are arranged in a manner such that the zigzag planes of the main chains are parallel to the $a^{\prime} c$ plane forming a sheet structure. In one sheet the $\mathrm{C}=\mathrm{O}$ groups of every chain orient in one direction ( $a$ or $-a$ direction), and in the neighboring sheet the chains orient oppositely. However, the chains are random in their levels in the fiber direction as shown schematically in Figure 6. It may be

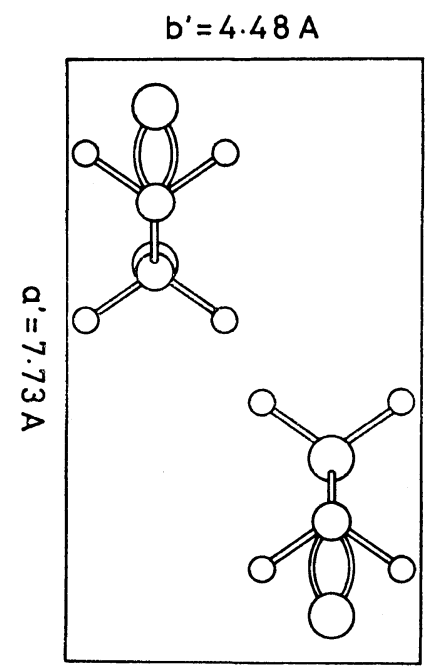

Figure 5. Molecular arrangement in Form II of poly( $\beta$-propiolactone) viewed along the fiber axis. 


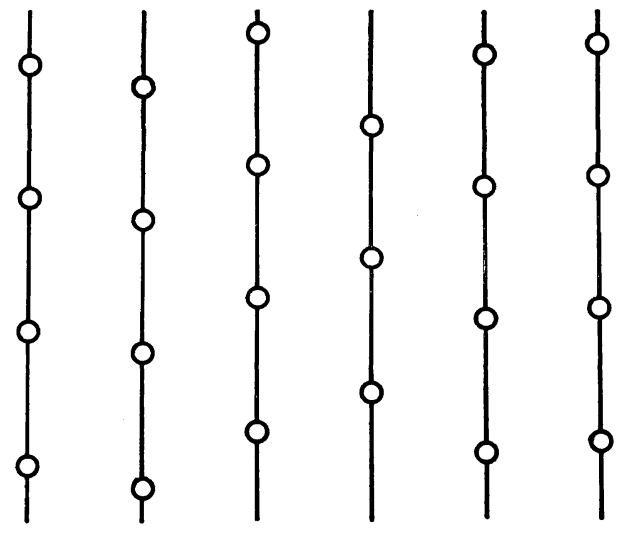

Figure 6. Schematic representation of molecular arrangement along the fiber axis. Lines represent molecular chains, and circles indicate carbonyl groups.

said that Form II is in a typical paracrystalline state. In spite of the paracrystalline nature, Form II seems to be very stable; Form II did not transform into other crystalline modifications even if it was annealed at near the melting point.

Following the paracrystal theory, ${ }^{11}$ the lattice factor for the present disordered structure can be expressed as follows;

$$
\begin{aligned}
& Z(h k l) \\
& =\frac{1-\exp \left(-4 \pi^{2} g_{13}^{2} l^{2}\right)}{1+\exp \left(-4 \pi^{2} g_{13}^{2} l^{2}\right)-2 \exp \left(-2 \pi^{2} g_{13}^{2} l^{2}\right) \cos 2 \pi h}
\end{aligned}
$$

$$
\times \frac{1-\exp \left(-4 \pi^{2} g_{23}^{2} l^{2}\right)}{1+\exp \left(-4 \pi^{2} g_{23}^{2} l^{2}\right)-2 \exp \left(-2 \pi^{2} g_{23}^{2} l^{2}\right) \cos 2 \pi k}
$$

In this expression, $h$ and $k$ are not always integers but any values, and $g_{13}$ and $g_{23}$ are the matrix elements of relative fluctuation of the lattice vectors $a^{\prime}$ and $b^{\prime}$ in the $c$ direction. It can be shown that the intensity distributions on the layer lines are smooth when $g_{13}$ and $g_{23}$ are greater than about 0.35 . However, it is difficult to argue the detail on the anisotropic feature of the disorder along the $a$ axis $\left(g_{13}\right)$ and the $b$ axis $\left(g_{23}\right)$ from the uniaxially oriented fiber pattern.

From consideration of the molecular structure and the molecular packing, such a disorder should be attributed to existence of some stable sites about the chain level in the fiber direction. The molecular chain has two senses with respect to the chain direction, that is, up and down. Therefore it is a possibility that chains with up and down senses locate at one site. The detail of the disorder in Form II and the structures of other modifications will be revealed in the near future.

Small angle X-ray diffraction patterns of fibrous Form II samples prepared by the ionic polymerization and the solid state polymerization are shown in Figure 7. A melt-cold drawn sample obtained by the ionic polymerization gave a

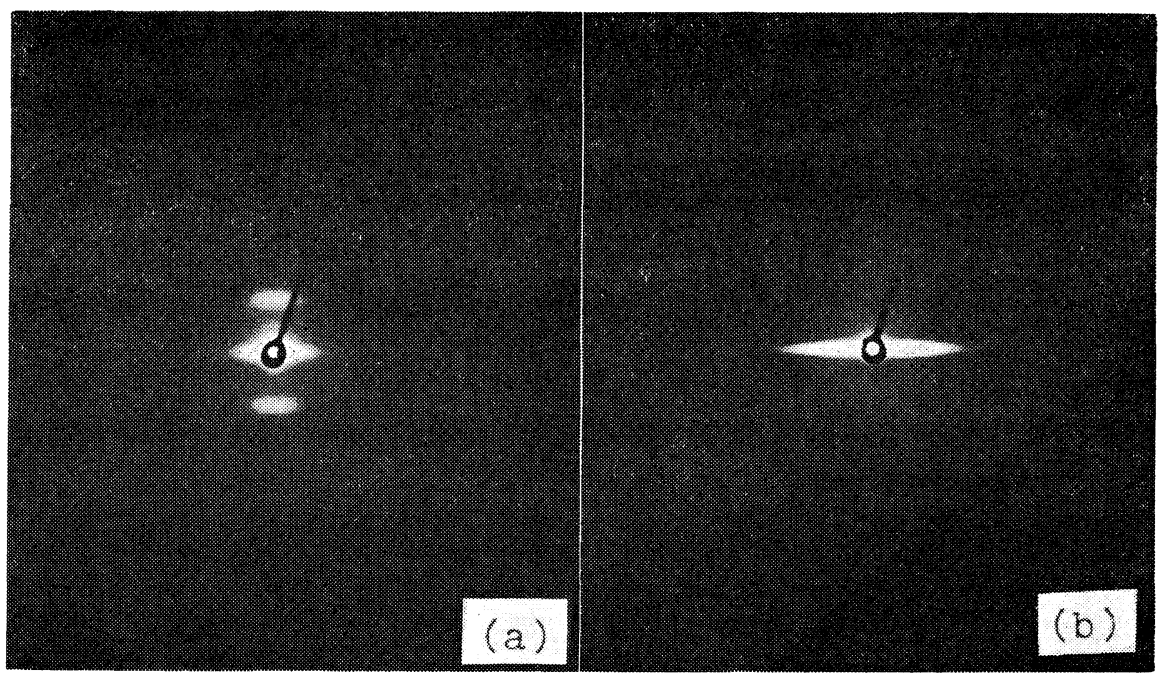

Figure 7. Small-angle X-ray photographs of Form II of poly( $\beta$-propiolactone): (a), melt-cold drawn sample; (b), as-polymerized sample by the solid-state polymerization. 
meridional reflection with a long spacing of $76 \AA$, according to Bragg's law, along the fiber axis. It is interesting that such a long periodic structure exists in the disordered state which might reflect a lamellar structure. On the other hand, no meridional reflection was observed in the aspolymerized sample obtained by the solid state polymerization. Since it is likely that the polymerization and crystallization of polymer in the solid state polymerization take place under a restricted environment, it is not surprising that the fine structure of the as-polymerized polymer is different from that of the melt or solutiongrown crystallized polymer. Similar behavior was found in the case of solid state polymerization of the cyclic oligomers of formaldehyde ${ }^{12}$ and the canal polymerization of 2,3-dimethylbutadiene and 2,3-dichlorobutadiene in the thiourea adducts. ${ }^{13}$

The melting point of the as-polymerized polymer formed by the solid state polymerization was $120^{\circ} \mathrm{C}$, while that of the melt-cold drawn polymer obtained by the ionic polymerization was $80-81^{\circ} \mathrm{C}$. If the as-polymerized polymer was subjected once to a melt-cold drawing treatment, the melting point dropped to $82^{\circ} \mathrm{C}$. Therefore, this behavior also supports the assumption that the as-polymerized polymer is different from the melt-cold drawn sample in fine structure.

Acknowledgment. The authors wish to express their sincere thanks to Professor S. Okamura of Kyoto University for his kind discussion and supplying the solid-state polymerized sample. The authors also wish to thank Daisel Co. Ltd. for the supply of the ionic polymerized sample.

\section{APPENDIX}

The intensity formula for X-ray diffraction by matter having a two dimensional lattice imperfection with no correlation between cells is written in the form, ${ }^{14}$

$$
\begin{aligned}
I(\boldsymbol{S})= & \frac{\sin ^{2} \pi N_{3} s_{3} c}{\sin ^{2} \pi s_{3} c}\left[|\langle F(\boldsymbol{S})\rangle|^{2} \frac{\sin ^{2} \pi N_{1} s_{1} a}{\sin ^{2} \pi s_{1} a}\right. \\
& \left.\times \frac{\sin ^{2} \pi N_{2} s_{2} b}{\sin ^{2} \pi s_{2} b}+N_{1} N_{2}\left\{\left\langle|F(\boldsymbol{S})|^{2}\right\rangle-|\langle F(\boldsymbol{S})\rangle|^{2}\right\}\right]
\end{aligned}
$$

where $\left\langle>\right.$ means average. $N_{1}, N_{2}$, and $N_{3}$ are the number of repeats along the $a, b$, and $c$ axes, respectively, and $s_{1}, s_{2}$, and $s_{3}$ are the components of the reciprocal lattice vector $S$. When the imperfection is restricted to the mutual level of molecular chains in the $c$ direction, the structure factor is given by

$$
F(\boldsymbol{S})=\sum_{m} F_{m}(\boldsymbol{S}) \exp \left(2 \pi \mathrm{i} s_{3} z_{m}^{\prime}\right)
$$

where $F_{m}(\boldsymbol{S})$ is the structure factor of the $m$ th chain and $z_{m}^{\prime}$ is the standard $z$ coordinate of the $m$ th chain.

If random displacement of the chains along the $c$ axis is assumed,

$$
\langle F(\boldsymbol{S})\rangle=\sum_{m} F_{m}(\boldsymbol{S}) \int_{0}^{c} \exp \left(2 \pi \mathrm{i} s_{3} z_{m}^{\prime}\right) \mathrm{d} z_{m}^{\prime} / c
$$

and

$$
\begin{aligned}
\left\langle|F(\boldsymbol{S})|^{2}\right\rangle= & \sum_{m m^{\prime}} F_{m}(\boldsymbol{S}) F_{m^{\prime}}^{*}(\boldsymbol{S}) \\
& \times \int_{0}^{c} \int_{0}^{c} \exp \left[2 \pi \mathrm{i} s_{3}\left(z_{m}^{\prime}-z_{m^{\prime}}^{\prime}\right)\right] \frac{\mathrm{d} z_{m}^{\prime}}{c} \frac{\mathrm{d} z_{m^{\prime}}^{\prime}}{c}
\end{aligned}
$$

Equations A3 and A4 become for $s_{3}=0$

$$
\begin{gathered}
\langle F(\boldsymbol{S})\rangle=\sum_{m} F_{m}(\boldsymbol{S}) \\
\left\langle|F(\boldsymbol{S})|^{2}\right\rangle=\left|\sum_{m} F_{m}(\boldsymbol{S})\right|^{2}
\end{gathered}
$$

and for $s_{3} \neq 0$

$$
\begin{aligned}
\langle F(\boldsymbol{S})\rangle & =\frac{\sin \pi s_{3} c}{\pi s_{3} c} \exp \left(\pi \mathrm{i} s_{3} c\right) \sum_{m} F_{m}(\boldsymbol{S}) \\
\left\langle|F(\boldsymbol{S})|^{2}\right\rangle= & \frac{\sin ^{2} \pi s_{3} c}{\left(\pi s_{3} c\right)^{2}}\left|\sum_{m} F_{m}(\boldsymbol{S})\right|^{2} \\
& +\left\{1-\frac{\sin ^{2} \pi s_{3} c}{\left(\pi s_{3} c\right)^{2}}\right\} \sum_{m}\left|F_{m}(\boldsymbol{S})\right|^{2}
\end{aligned}
$$

Here on substituting $s_{3}=0$, eq $\mathrm{A} 6 \mathrm{a}$ and $\mathrm{A} 6 \mathrm{~b}$ are reduced to eq $\mathrm{A} 5 \mathrm{a}$ and $\mathrm{A} 5 \mathrm{~b}$, respectively. Therefore, the intensity formula is expressed by eq A7.

$$
\begin{aligned}
I(\boldsymbol{S})= & {\left[\left|\sum_{m} F_{m}(\boldsymbol{S})\right|^{2} \frac{\sin ^{2} \pi N_{1} s_{1} a}{\sin ^{2} \pi s_{1} a} \frac{\sin ^{2} \pi N_{2} s_{2} b}{\sin ^{2} \pi s_{2} b}\right.} \\
& \times \frac{\sin ^{2} \pi s_{3} c}{\left(\pi s_{3} c\right)^{2}}+N_{1} N_{2} \sum_{m}\left|F_{m}(\boldsymbol{S})\right|^{2} \\
& \left.\times\left\{1-\frac{\sin ^{2} \pi s_{3} c}{\left(\pi s_{3} c\right)^{2}}\right\}\right] \frac{\sin ^{2} \pi N_{3} s_{3} c}{\sin ^{2} \pi s_{3} c}
\end{aligned}
$$




\section{REFERENCES}

1. A.S. Ueda, Y. Chatani, and H. Tadokoro, Polymer J., 2, 387 (1971).

2. R. K. Hasegawa, Y. Chatani, and H. Tadokoro, ibid., to be published.

3. Y. Chatani, K. Suehiro, Y. Okita, H. Tadokoro, and K. Chujo, Makromol. Chem., 113, 215 (1968).

4. M. Yokouchi, Y. Chatani, H. Tadokoro, K. Teranishi, and H. Tani, Polymer, 14, 267 (1973).

5. M. Yokouchi, Y. Chatani, H. Tadokoro, and H. Tani, Polymer J., 6, 248 (1974).

6. A. Turner-Jones and C. W. Bunn, Acta Crystallogr., 15, 105 (1962).

7. Y. Chatani, Y. Okita, H. Tadokoro, and Y. Yamashita, Polymer J., 1, 555 (1970).

8. S. Okamura, T. Higashimura, A. Tanaka, R.
Kato, and Y. Kikuchi, Macromol. Chem., 54, 226 (1962).

9. K. Suehiro, unpublished data.

10. T. Wasai, T. Saegusa, and J. Furukawa, Kogyo Kagaku Zasshi (J. Chem. Soc. Japan, Ind. Chem. Sect.), 67, 601 (1964).

11. R. Hosemann and S. N. Bagchi, "Direct Analysis of Diffraction by Matter," North-Holland, 1962.

12. Y. Chatani, T. Uchida, H. Tadokoro, K. Hayashi, M. Nishii, and S. Okamura, J. Macromol. Sci.-Phys., B2, 567 (1968).

13. Y. Chatani and S. Nakatani, Macromolecules, 5, 597 (1972).

14. I. Kakinoki, "X-Sen Kesshogaku (X-ray Crystallography)," Vol. II, I. Nitta, Ed., Maruzen, Tokyo, 1961, p 653. 